36 weeks GA increased significantly in infants 1,000-1,500 g and head circumference at 36 weeks GA increased significantly in all infants. No significant differences were seen in the rates of NEC, BPD, ROP, IVH and PVL.

Conclusions Modified nutritional protocol based on supplying the early aggressive macronutrients and higher calorie, can significantly reduce the incidence of EUGR in infants $\leq 1,500 \mathrm{~g}$ without any complications. We need further investigation to improve growth in infants $<1,000 \mathrm{~g}$.

\section{PO-0588 NEONATAL OUTCOMES OF VERY LOW BIRTH WEIGHT INFANTS WHO RECEIVED ENTERAL NUTRITION WITH AND WITHOUT OLIVE OIL SUPPORT: RANDOMISED CONTROLLED PILOT STUDY}

${ }^{1}$ A Ecevit, ${ }^{1}$ A Abbasoglu, ${ }^{1} U$ Tugcu, ${ }^{1} M$ Silahli, ${ }^{2} Y$ Laleli, 'A Tarcan. ${ }^{1}$ Pediatrics Neonatology, Baskent University Medical School, Ankara, Turkey; ${ }^{2}$ Specialized Chemistry Division, Duzen Laboratory, Ankara, Turkey

\subsection{6/archdischild-2014-307384.1229}

Background and aims Very low birth weight (VLBW) infants have a greater risk for the oxidative stress related diseases (OSRDs) like retinopathy of prematurity, bronchopulmonary dysplasia, periventricular leukomalacia and necrotizing enterocolitis. Naturel antioxidant activity of phenols, flavonoid and tocopherols in extra virgin olive oil (EVOO) may be preventive for the OSRDs. The purpose of conducting a randomised controlled pilot study is to compare the weight gaining, length of hospitalisation and the OSRDs of VLBW infants who received early enteral nurtition with and without EVOO support.

Methods VLBW newborns were divided into two groups in this pilot study. Group 1 received enteral nutrition and EVOO, Group 2 received only enteral nutrition. Nutritional analysis was undertaken for EVOO that was added as $0.5 \mathrm{ml} /$ day in $100 \mathrm{ml}$ enteral nutrition. Total parenteral nutrition (TPN) and minimal enteral nutrition was initiated both of two groups.

Results A total of 26 VLBW infants were divided into two groups (Group 1)(n=13) and (Group 2) $(\mathrm{n}=13)$ and assessed the birth weight: Group $1=1,329 \pm 35$ g, Group $2=1,276 \pm$ 32 g. gestational age: Group $1=31 \pm 2.79$, Group $2=29 \pm 2$ weeks. There was no significant difference between two groups for weight gaining, length of hospitalisation and the OSRDs.

Conclusions EVOO is very important natural antioxidant and anti-inflammatory nutrients for preterm infants particularly VLBW. A larger randomised controlled trials are needed to show the antioxidant and anti-inflammatory effects of olive oil for prevention of OSRDs in this high risk group.

\section{PO-0589 A MIXED BAG - HAS THE NATIONAL CONFIDENTIAL ENQUIRY INTO PATIENT OUTCOME AND DEATH (NCEPOD-REPORT, UK 2010) MADE A DIFFERENCE TO THE NUTRITION OF PRETERM INFANTS?}

${ }^{1} \mathrm{E}$ Chan, ${ }^{2} \mathrm{C}$ Smith, ${ }^{2} \mathrm{C}$ Davidson, ${ }^{3} \mathrm{R}$ Watkins, ${ }^{3} \mathrm{P}$ Amess, ${ }^{3} \mathrm{H}$ Rabe, ${ }^{3} \mathrm{JR}$ Fernandez Alvarez. ${ }^{1}$ Neonatology, Brighton and Sussex Medical School, Brighton, UK; ${ }^{2}$ Paediatric Dietetics, Brighton and Sussex University Hospitals NHS Trust, Brighton, UK; ${ }^{3}$ Neonatology, Brighton and Sussex University Hospitals NHS Trust, Brighton, UK

\subsection{6/archdischild-2014-307384.1230}

Background Early parenteral nutrition (PN) improves growth in preterm neonates. Good practice in $\mathrm{PN}$-care was identified in only $24 \%$ of the units in the NCEPOD-Report.

\begin{tabular}{|c|c|c|c|c|}
\hline & & BWz & $\mathrm{DWz}$ & TPN \\
\hline & 23-30GA & $-0.59(-1.02 / 0.07)$ & $-2.18(-2.57 /-1.59)$ & $12(7-18)$ \\
\hline \multirow[t]{2}{*}{2010} & $31-36 \mathrm{GA}$ & $-0.28(-1.11 / 0.36)$ & $-1.46(-1.95 /-0.95)$ & $0(0-1)$ \\
\hline & 23-30GA & $-0.32(-1.17 / 0.34)$ & $-1.4(-2.03 /-1.0)$ & $11(7-13)$ \\
\hline \multirow[t]{2}{*}{2011} & $31-36 \mathrm{GA}$ & $-0.4(-1.33 / 0.11)$ & $-1.92(-2.47 /-1.27)$ & $0(0-6)$ \\
\hline & 23-30GA & $-0.19(-0.9 /-0.06)$ & $-2.08(-2.69 /-1.28)$ & $8(1-10)$ \\
\hline \multirow[t]{2}{*}{2012} & $31-36 \mathrm{GA}$ & $-0.92(-1.1 / 0.07)$ & $-2.19(-2.63 /-1.44)$ & 10(3-11) \\
\hline & $23-30 \mathrm{GA}$ & $-0.38(-1.63 / 0.07)$ & $-2.04(-2.7 /-1.73)$ & $6(0-13)$ \\
\hline 2013 & $31-36 \mathrm{GA}$ & $-0.75(-1.52 /-0.27)$ & $-1.98(-2.69 /-1.36)$ & $8(4-10)$ \\
\hline Term & & $-1.53(-2.24 /-0.34)$ & $-1.63(-2.7 /-0.91)$ & \\
\hline
\end{tabular}

Aim To compare the growth of preterm infants since the NCEPOD-Report in relation to PN-use.

Methods Retrospective comparison of preterm infants cared for in a tertiary neonatal unit until 36 weeks gestational age (GA) between 2010-2013. Newborns were grouped by gestation (2330GA/31-36GA) and birth-year. Outcomes were z-scores for birthweight $(\mathrm{BWz})$, discharge-weight $(\mathrm{DWz})$ and length of $\mathrm{PN}$ in days (loPN). Outcomes were compared between years, within years and between GA-groups including >36GA-control-group. Data-presentation/-analysis: Median (interquartile-range); MannWhitney-U-Test/Kruskal-Wallis-Test ( $\mathrm{p}<0.05$ ).

Results 175 newborns recruited. No significant difference for $\mathrm{BWz}$ and $\mathrm{DWz}$ between years and for $\mathrm{BWz}$ and $\mathrm{DWz}$ between GA-groups within a year except for DWz 2010(p = 0.02). No significant difference for the same GA-group between years except for DWz 23-30GA(p = 0.04). No significant difference for loPN between years and between GA-groups within a year except for $2010(\mathrm{p}<0.0001)$ and $2011(\mathrm{p}<0.0001)$. No significant difference for the same GA-group between years except for $31-36 \mathrm{GA}(\mathrm{p}<0.0001)$.

Conclusion Growth in preterm infants assessed by difference in z-scores appears to have improved since 2010. This may be partly due to increased PN-use which although not significant shows a notable increase since the NCEPOD-Report.

\section{P0-0590 SUBSTANCE ABUSE DETERMINATION IN ALTERNATIVE MATRICES OF BREASTMILK DONORS}

${ }^{1} \mathrm{~N}$ García-Lara, ${ }^{2} \mathrm{D}$ Escuder-Vieco, ${ }^{3} \mathrm{O}$ García-Algar, ${ }^{4} \mathrm{E}$ Marchei, ${ }^{4} \mathrm{~S}$ Pichini, ${ }^{2}$ CR Pallás-Alonso. ${ }^{1}$ Neonatology, Hospital 12 de Octubre, Madrid, Spain; ${ }^{2}$ Neonatology, Hospital 12 de Octubre, Madrid, Spain; ${ }^{3}$ Unitat de Recerca Infància i Entorn, IMIM-Hospital Del Mar, Barcelona, Spain; ${ }^{4}$ Instituto Superiore di Sanita, Drug Control and Evaluation Department, Roma, Italy

\subsection{6/archdischild-2014-307384.1231}

Background and aims In human milk banks, the only available information regarding toxicological safety, is provided by donors in the screening questionnaire. A good agreement between donors' self-report and milk analysis for nicotine and illegal drug use (excluding caffeine) was reported. Determination of these substances in urine and hair samples may provide additional information.

Our main objetive was to determine drug abuse substances, nicotine and caffeine, in donors' breastmilk, urine and hair and compare the results to donors' answers in the screening questionnaire.

Methods 36 samples of breastmilk, urine and hair from 36 milk donors were collected. All donors completed a lifestyle questionnaire. A validated, reversed-phase liquid chromatography tandem 